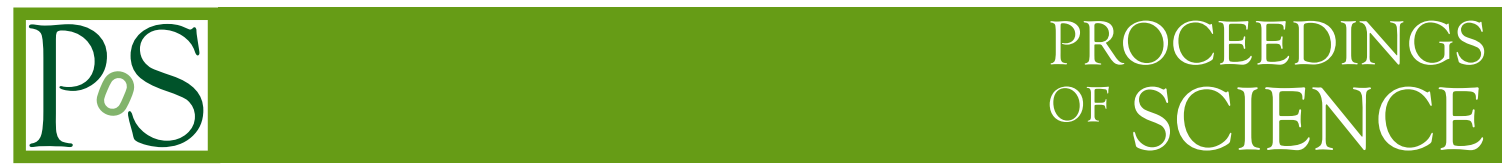

\title{
Searches for Higgs boson rare and invisible decays at CMS
}

\author{
Vukašin Milošević ${ }^{a, b, *}$ on behalf of the CMS Collaboration \\ ${ }^{a}$ Imperial College London, \\ Prince Consort Road, London, UK \\ ${ }^{b}$ University of Belgrade, Faculty of Physics, \\ Studentski trg 12, Belgrade, Serbia \\ E-mail: vukasin.milosevic@cern.ch
}

The latest results of searches for the invisible decays of the Higgs boson $(\mathrm{H} \rightarrow$ inv $)$ performed by the CMS Collaboration are presented. These studies represent a combination effort focusing on the data collected during the Run $1(\sqrt{s}=7$ and $8 \mathrm{TeV})$ and the early part of Run $2(\sqrt{s}=13 \mathrm{TeV})$ period within the Large Hadron Collider (LHC). The combined result is expressed as a $95 \%$ Confidence Level (CL) upper limit on the branching fraction and currently stands at $\mathcal{B}(\mathrm{H} \rightarrow \mathrm{inv})<0.19(0.15)$ for the observed (expected) limit. Studies of the Higgs boson decaying to a dimuon pair $(\mathrm{H} \rightarrow \mu \mu)$ are also presented. This result takes advantage of the complete dataset collected during the Run 2 period and constitutes the first evidence for the $\mathrm{H} \rightarrow \mu \mu$ decay with the observed (expected) significance of 3.0 (2.5) $\sigma$ and the measured signal strength, relative to the Standard Model (SM), of $\mu=1.19_{-0.39}^{+0.41}$ (stat) ${ }_{-0.16}^{+0.17}$ (sys).

40th International Conference on High Energy physics - ICHEP2020

July 28 - August 6, 2020

Prague, Czech Republic (virtual meeting)

\footnotetext{
${ }^{*}$ Speaker
} 


\section{Introduction}

Following the discovery of the Higgs boson by the ATLAS and CMS Collaborations [1, 2], a set of precision measurements has been launched in order to test if the properties of the newly discovered particle agree well with the SM. As it currently stands, all of the measurements have shown a good agreement with the SM. The uncertainties on these measurements, however, can allow for physics beyond the SM (BSM). According to the SM, a decay mode that would prove to be invisible to current detectors at the $\mathrm{LHC}$ is the $\mathrm{H} \rightarrow \mathrm{ZZ} \rightarrow 4 v$ decay, which has a predicted branching fraction of $\mathcal{B}(\mathrm{H} \rightarrow 4 v) \approx 0.1 \%$. This makes the invisible final state an interesting channel for probing for new physics at the LHC. On the side of precision studies of the SM, the dimuon final state represents a good channel for probing the Higgs boson coupling to the second generation of fermions. It is a challenging process to study, due to its small branching fraction of $\mathcal{B}(\mathrm{H} \rightarrow \mu \mu) \approx 2.19 \cdot 10^{-4}$. The following sections summarise both of these topics, in the form of their latest results, from the perspective of the CMS experiment [3].

\section{Invisible decays of the Higgs boson}

The main idea behind the BSM approach for the invisible final state is that the Higgs boson can take a role of a mediator connecting the SM particles with the ones originating from the dark matter sector. The process of detection for this signature requires that the Higgs boson recoils against a visible system, while the invisible contribution is to be quantified through the appearance of a large value of the missing transverse energy $\left(E_{\mathrm{T}, \mathrm{miss}}\right)^{1}$.

The analysis channel with the most sensitivity towards the invisible final state is formed around the properties of the vector boson fusion (VBF) production mode of the Higgs boson. The main characteristics of the VBF channel are the appearance of two energetic jets with a large geometrical separation, followed by a large dijet invariant mass. The analysis strategy includes selection requirements based on the aforementioned properties of VBF jets as well as the use of data driven methods to constrain the contribution of main irreducible SM backgrounds, such as the production of $\mathrm{W}$ and $\mathrm{Z}$ bosons, in association with two jets, subsequently decaying to neutrinos (where, in the former case, a final state lepton gets lost in the detection process). The next mode of interest is the production of the Higgs boson in association with a vector boson $(\mathrm{VH})$. The analysis of this production mode is further split into two categories, basing the separation on the decay of the accompanying vector boson. The scenario where the vector boson further decays into energetic jets forms the $\mathrm{V}\left(\mathrm{qq}^{\prime}\right) \mathrm{H}$ category, while the scenario where the $\mathrm{Z}$ boson decays leptonicaly forms the $\mathrm{Z}(11) \mathrm{H}$ category. The final analysis channel of interest focuses on the gluon-gluon fusion production of the Higgs boson $(\mathrm{ggH})$. Its final state requires a jet from initial-state radiation ${ }^{2}$.

The latest published combination effort summarises results from previously introduced analysis channels and uses the data collected during the entire Run 1 and early Run 2 periods of LHC data collection [4]. This includes the total integrated luminosity of $4.9 \mathrm{fb}^{-1}(\sqrt{s}=7 \mathrm{TeV})$ and $19.7 \mathrm{fb}^{-1}$ $(\sqrt{s}=8 \mathrm{TeV})$ from the Run 1 and $38.2 \mathrm{fb}^{-1}(\sqrt{s}=13 \mathrm{TeV})$ from the 2015 and 2016 eras of

\footnotetext{
${ }^{1}$ Defined as the magnitude of the negative sum of transverse momenta of all visible particles, or: $E_{\mathrm{T}, \mathrm{miss}}=\left|-\sum_{i} \vec{p}_{\mathrm{T}, i}\right|$

${ }^{2}$ There is a significant contribution to the VBF channel originating from the ggH production, where it populates low values of the dijet invariant mass.
} 
data collection. Figure 1 (left) summarises the $95 \% \mathrm{CL}$ upper limits on the $\left(\sigma / \sigma_{S M}\right) \mathcal{B}(\mathrm{H} \rightarrow \mathrm{inv})$ placed from the perspective of studies focusing on the 2016 data (with $35.9 \mathrm{fb}^{-1}$ of total integrated luminosity). It shows the largest sensitivity brought by the VBF channel, by placing the observed (expected) limit on $\mathcal{B}(\mathrm{H} \rightarrow \mathrm{inv})^{3}$ at $0.33(0.25)$. The $\mathrm{Z}(11) \mathrm{H}$ channel places the limit at $0.40(0.42)$, while the $\mathrm{V}\left(\mathrm{qq}^{\prime}\right)$ yields a value of $0.50(0.48)$. Lastly, the ggH channel sets a limit on $\mathcal{B}(\mathrm{H} \rightarrow \mathrm{inv})$ at $0.66(0.59)$. The combination of the aforementioned measurements places a limit on $\mathcal{B}(\mathrm{H} \rightarrow \mathrm{inv})$ at $0.26(0.20)$. These measurements were combined with the results coming from Run 1 studies, as shown in Figure 1 (right), ultimately setting the limit at $0.19(0.15)$.

An update on these studies is currently being prepared with the focus being placed on the analysis of the full Run 2 dataset. The first combined Run 2 result (performed on the $137 \mathrm{fb}^{-1}$ ) focusing on the $\mathrm{Z}(11) \mathrm{H}$ channel places an observed (expected) $95 \% \mathrm{CL}$ upper limit on $\mathcal{B}(\mathrm{H} \rightarrow \mathrm{inv})$ at $0.29(0.25)$ [5]. Studies focusing on other channels are expected to provide updated results in the coming months.
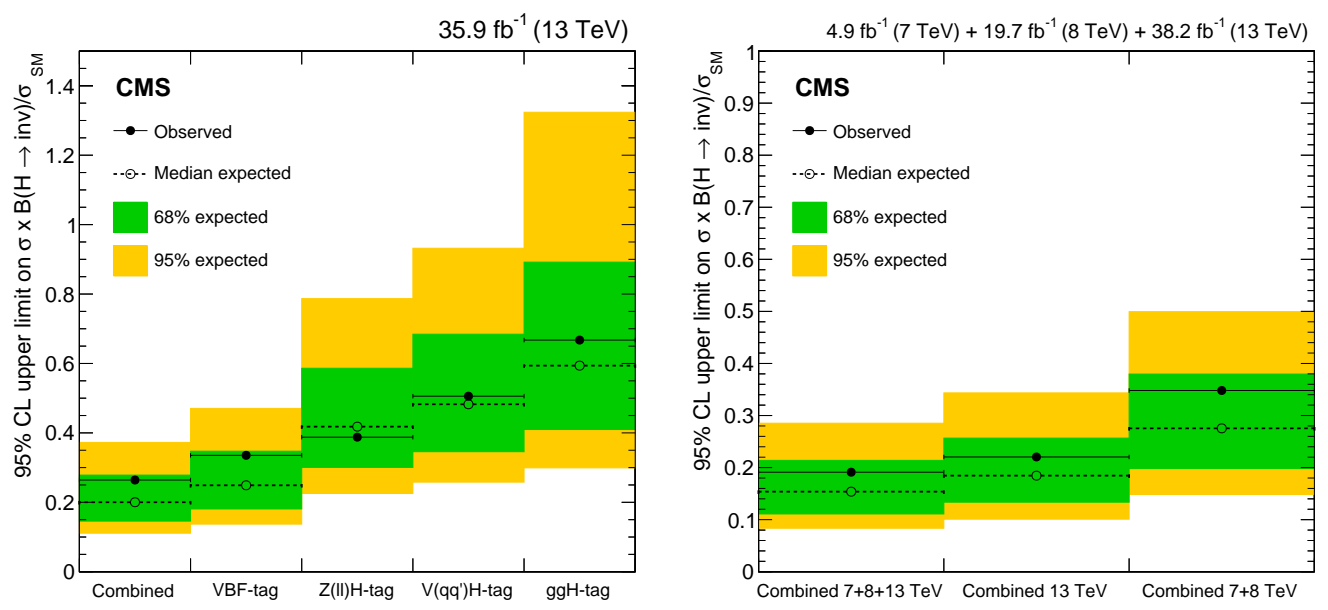

Figure 1: Summary of values for the $95 \% \mathrm{CL}$ upper limits on the $\left(\sigma / \sigma_{S M}\right) \mathcal{B}(\mathrm{H} \rightarrow$ inv $)$ for the measurement focusing on the 2016 dataset (left), split into separate channels and a combined result [4]. The subsequent combination with previous Run 1 results (right). [4].

\section{Higgs boson decay to a pair of muons}

The latest study of the dimuon decay of the Higgs boson constitutes the first evidence for this process, obtained by analysing the complete Run 2 dataset $\left(\sqrt{s}=13 \mathrm{TeV}\right.$, with $137 \mathrm{fb}^{-1}$ of total integrated luminosity) [6]. The analysis strategy splits the interesting phase space into four mutually exclusive channels, based on the production mode of the Higgs boson. A common requirement for all channels is the request for a narrow dimuon invariant mass $\left(m_{\mu \mu}\right)$ range around the Higgs boson mass: $110<m_{\mu \mu}<150 \mathrm{GeV}$. This region is further split into the mass sideband region (SB): $110<m_{\mu \mu}<115 \mathrm{GeV}$ or $135<m_{\mu \mu}<150 \mathrm{GeV}$, used to study the background dominated phase space of interest, and the mass signal region (SR): $115<m_{\mu \mu}<135 \mathrm{GeV}$.

\footnotetext{
${ }^{3}$ Under the assumption of the SM cross section for the respective production mode.
} 
Categorisation for this final state was done following a similar idea to the previously described $\mathrm{H} \rightarrow \mathrm{inv}$ study, by focusing around different production modes of the Higgs boson. The VBF channel explores the properties of the associated dijet topology alongside the muon requirements. The main background for this category is SM productions of $\mathrm{Z}$ boson in association with two jets. The second channel of interest is based on the Higgs production associated with top quarks (ttH). It is focused around a production mode with the smallest cross section, but the presence of top quarks provides a valuable signature for background rejection. Events falling under this channel can further be separated into two categories, based on the final state of the $\mathrm{W}$ boson originating from the $t \rightarrow b W$ decay. The first scenario is the leptonic decay where the final state can include one or two additional leptons. For this, multiple lepton, scenario, the choice of the muons originating from the Higgs boson decay is made by selecting those with the largest value of transverse momenta. Main sources of background for this leptonic subcategory are represented with tt and ttZ processes. The other scenario focused on the fully hadronic final state of the $\mathrm{W}$ boson decay, being confronted with tt processes as its main source of SM background. The next channel, targeting VH production, focused on searching for additional leptons, besides the dimuon pair, and is further split into $\mathrm{ZH}$ ( $4 \mu$ or $2 \mu 2 e$ final state) and $\mathrm{WH}$ ( $3 \mu$ or $2 \mu 1 e$ final state). Lastly, the ggH channel, collects all events discarded by other categories, with the dominating background being the Drell-Yan process.

The analysis strategy for the ggH category employs a boosted decision tree (BDT) multivariate discriminator. It is trained through the use of muon variables, while being kept largely uncorrelated with $m_{\mu \mu}$. The BDT discriminator has been trained separately for each data taking period in order to account for different detector effects during different eras. Further division of selected events into categories was done iteratively, being based on the S/B criteria. Significance is then extracted through a fit of the $m_{\mu \mu}$ variable using analytical functions which are inspired from simulation (following from the fact that both signal and background compositions are stable across all ggH subcategories). Each production mode of the signal was modelled with a Double Crystal-ball function, while the background modelling was done through the use of functions in the discrete envelope (Breit-Wigner, etc.) which are being modulated in each category via polynomials. This strategy is followed by $\mathrm{VH}$ and $\mathrm{ttH}$ categories. For the $\mathrm{VH}$ categories, BDTs are trained to explore the $\mathrm{Z}$ or $\mathrm{W}$ topology as well as the dimuon signature. In this case, backgrounds are being modelled via modified Breit-Wigner functions. Similarly, the BDTs deployed within the $\mathrm{ttH}$ categories are trained using both $\mu \mu$ and tt variables (while still keeping the BDT output uncorrelated with $m_{\mu \mu}$ ). For this group of categories, a set of polynomial and exponential functions is used to fit the background. Lastly, the VBF category, due to the limited statistical precision of the mass $\mathrm{SB}$ region, relies on the use of simulated samples for the purpose of estimating the contribution of main SM backgrounds. A deep neural network (DNN) multivariate discriminator is used to distinguish signal over background (with the training being performed in both SR and SB regions). A maximum likelihood fit to the DNN output score is being performed simultaneously in the SR and SB, independently per data taking period. This inclusion of the SB into the fit further constrains the background normalisation.

A simultaneous fit was performed across all four channels, with the signal strength being defined as $\mu=\left[\sigma \mathcal{B}(H \rightarrow \mu \mu)_{o b s}\right] /\left[\sigma \mathcal{B}(H \rightarrow \mu \mu)_{S M}\right]$. Expressed for the Higgs boson mass of $m_{H}=125.38 \mathrm{GeV}$ [7], the signal strength is measured to be: $\mu=1.19_{-0.39}^{+0.41}$ (stat $)_{-0.16}^{+0.17}($ syst $)$. This measurement marks the first evidence for the dimuon decay of the Higgs boson, by reporting 
an excess with a 3.0 (2.5) $\sigma$ observed (expected) significance. The left side of Figure 2 shows the $\mathrm{S} /(\mathrm{S}+\mathrm{B})$ weighted $m_{\mu \mu}$ distribution, combining all channels for illustration purposes (it does not represent the fit results), while the right side summarises the per-channel measured values of the signal strength. Lastly, a combination with studies focusing on the Run 1 period has been performed. Figure 3 shows a comparison of Higgs coupling parameter values expressed in terms of the particle mass for the latest, combined Run 2, measurement.
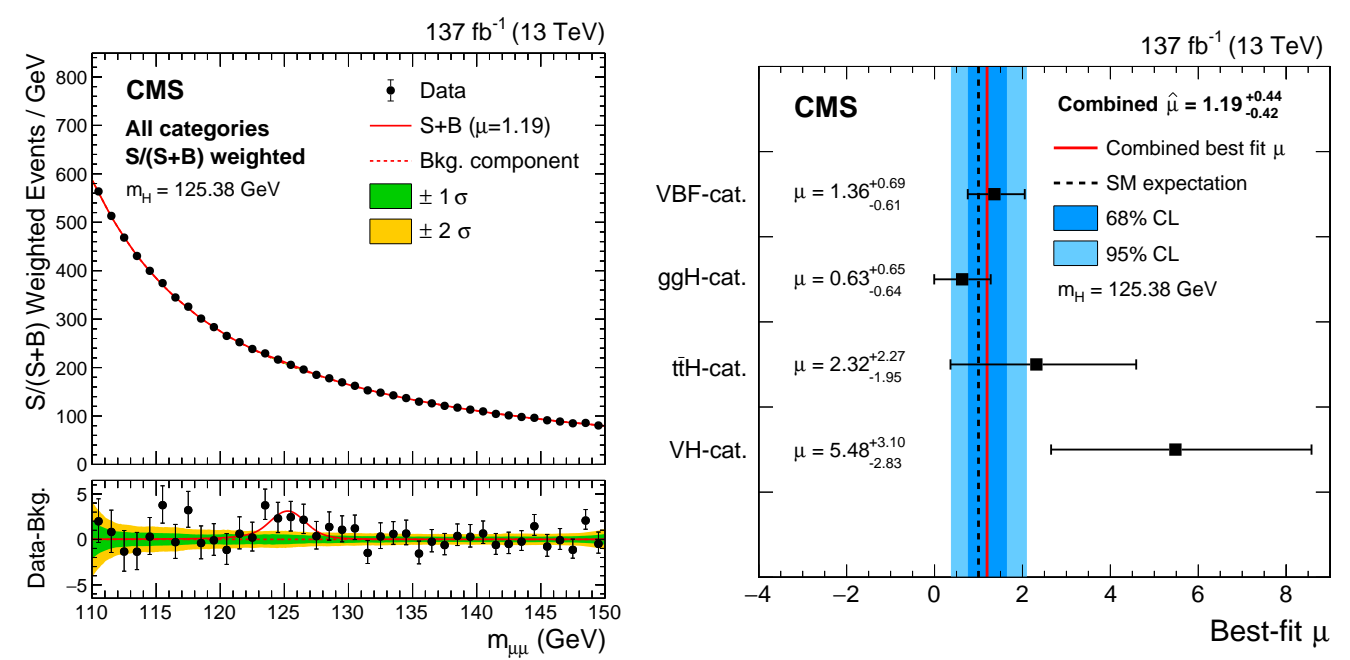

Figure 2: Summary of all analysis categories represented in terms of the $m_{\mu \mu}$ (left) [6]. Values of the measured signal strength for each of the main analysis channels (right) [6].

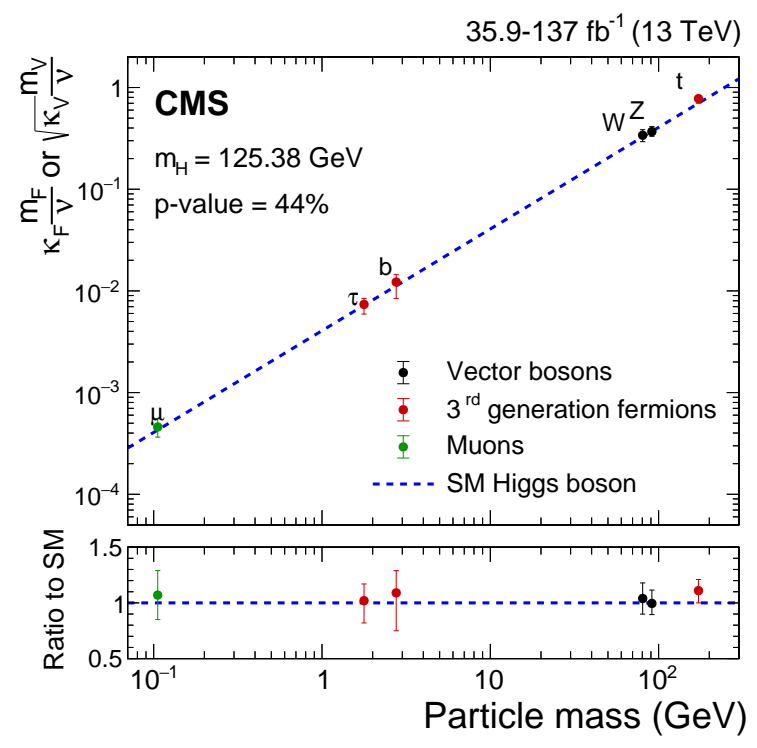

Figure 3: The Higgs coupling parameter values presented for different particle masses summarising measurements using the latest, complete Run 2, measurement [6]. 


\section{Conclusion}

This document summarised the most recent results involving rare and invisible decays of the Higgs boson from the perspective of the CMS experiment. The latest combination effort of different studies targeting the invisible final state, focusing on the Run 1 and 2015-2016 periods of data collection, have been presented. This effort places an observed (expected) $95 \%$ CL upper limit on the $\mathcal{B}(\mathrm{H} \rightarrow \mathrm{inv})$ of $0.19(0.15)$. The study of the complete Run 2 dataset is ongoing and updated results are expected in the near future. The latest measurement targeting the $\mathrm{H} \rightarrow \mu \mu$ final state was also presented ( $\sqrt{s}=13 \mathrm{TeV}$, with $137 \mathrm{fb}^{-1}$ of total integrated luminosity). This analysis targets the four main Higgs production modes ( $g g H, V B F, ~ V H$ and ttH). An excess of events over the background expectation is observed (expected) with a significance of 3.0 (2.5) standard deviations and represents the first evidence for the Higgs boson coupling to the second generation.

\section{References}

[1] ATLAS Collaboration, "Observation of a new particle in the search for the Standard Model Higgs boson with the ATLAS detector at the LHC", Phys. Lett. B 716 (2012) 1

[2] CMS Collaboration, "Observation of a new boson at a mass of $125 \mathrm{GeV}$ with the CMS experiment at the LHC", Phys. Lett. B 716 (2012) 30

[3] CMS Collaboration, "The CMS experiment at the CERN LHC ", JINST 3 S08004 (2008)

[4] CMS Collaboration, "Search for invisible decays of a Higgs boson produced through vector boson fusion in proton-proton collisions at $\sqrt{s}=13$ TeV", Phys. Lett. B 793 (2019) 520

[5] CMS Collaboration "Search for dark matter produced in association with a leptonically

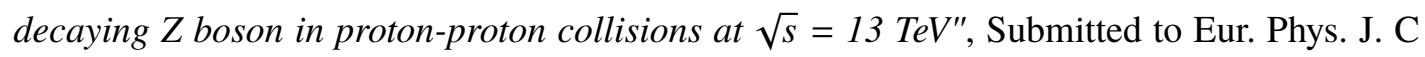
(2020)

[6] CMS Collaboration Evidence for Higgs boson decay to a pair of muons, Submitted to JHEP (2020)

[7] CMS Collaboration, A measurement of the Higgs boson mass in the diphoton decay channel, Phys. Lett. B 805 (2020) 135425

[8] CMS Collaboration, Combined measurements of Higgs boson couplings in proton-proton collisions at $\sqrt{s}=13 \mathrm{TeV}$, Eur. Phys. J. C 79 (2019) 421 